\title{
STABILITY OF SPIKY SOLUTIONS IN A REACTION-DIFFUSION SYSTEM WITH FOUR MORPHOGENS ON THE REAL LINE
}

\author{
JUNCHENG WEI * AND MATTHIAS WINTER ${ }^{\dagger}$
}

\begin{abstract}
We study a reaction-diffusion system with four morphogens which has been suggested in [23]. This system is a generalization of the Gray-Scott model $[10,11]$ and allows for multiple activators and multiple substrates. We construct single-spike solutions on the real line and establish their stability properties in terms of conditions of connection matrices which describe the interaction of the components. We use a rigorous analysis for the linearized operator around single-spike solutions based on nonlocal eigenvalue problems and generalized hypergeometric functions.

The following results are established for two activators and two substrates: Spiky solutions may be stable or unstable, depending on the type and strength of the interaction of the morphogens. In particular, it is shown that these patterns are stabilized in the following two cases: Case 1: interaction of different activators with each other (off-diagonal interaction of activators). Case 2: variation in strength of interaction of activators with different substrates (e.g. each activator has its preferred substrate).
\end{abstract}

Key words. Pattern Formation, Stability, Spike Solutions, Reaction-Diffusion System, Four Morphogens

AMS subject classifications. Primary 35B35, 92C40; Secondary 35B40

1. Introduction. We consider a reaction-diffusion system with many morphogens introduced by Takagi and Kaneko in [23] which is a generalization of the Gray-Scott model $[10,11]$ to many components. The generalized model in [23] considers the interaction of $N$ activators and $M$ substrates for any positive integers $N$ and $M$, whereas the Gray-Scott model only includes interactions of one activator and one substrate, i.e. the special case $M=N=1$. It is also a generalization of the hypercycle of Eigen and Schuster $[6,7,8,9]$ from one substrate (i.e. $M=1$ and $N$ any positive integer) to many substrates.

The interaction between these components is modeled by nonnegative connection matrices $W_{i}^{(j, k)}$, where the subscript $i=1, \ldots, M$ refers to one substrate and the superscripts $j, k=1, \ldots, N$ represent two activators. Any combination of $i, j, k$ is allowed. In particular, it is possible to have $j=k$ (self-interaction of an activator with itself) or $j \neq k$ (cross-interaction of different activators with each other), where each of these is mediated by a substrate.

The system can be written as follows:

(1.1) $\left\{\begin{array}{l}\tau \frac{\partial u_{i}}{\partial t}=D \Delta u_{i}+1-u_{i}-\frac{A}{\epsilon} u_{i} \sum_{j, k} W_{i}^{(j, k)} v_{j} v_{k}, i=1,2, \ldots, M, x \in \mathbb{R}, \\ \frac{\partial v_{i}}{\partial t}=\epsilon^{2} \Delta v_{i}-v_{i}+v_{i} \sum_{j, k=1} W_{j}^{(i, k)} u_{j} v_{k}, i=1,2, \ldots, N, x \in \mathbb{R}\end{array}\right.$

where $u_{i}$ and $v_{i}$ denote the concentrations of the substrates and the activators, respectively. Here $0<\epsilon^{2} \ll 1$ and $0<D$ are two positive diffusion constants. The

\footnotetext{
*Department of Mathematics, Chinese University of Hong Kong, Shatin, Hong Kong (wei@math. cuhk.edu.hk).

${ }^{\dagger}$ Brunel University, Department of Mathematical Sciences, Uxbridge UB8 3PH, United Kingdom (matthias.winter@brunel.ac.uk)
} 
constants $A$ (positive) and $\tau$ (nonnegative) will be treated as parameters since their choice will distinguish between stability and instability.

We will consider the special case $M=N=2$. This restriction is made in order to obtain explicit conditions for stability or instability which can be linked to biological applications. Some parts of the analysis are valid for general positive integers $M$ and $N$. This will be explained in some remarks below.

We first prove the existence of solutions with a steady-state single spike for which the activators have the same location and amplitude and the substrates have the same values at the position of the spike.

Then we investigate the linearized stability of these steady states. We study the linearized operator around spiky solutions using nonlocal eigenvalue problems and generalized hypergeometric functions. We will show that the spike may be stable or unstable and give conditions for both.

These results will be a generalization of properties for the Gray-Scott system. Let us briefly recall some previous papers on this issue: In $[2,3,4,5]$ the existence and stability of spike patterns on the real line is proved. In $[13,14]$ different regimes for the Gray-Scott systems are considered and the existence and stability of spike patterns in an interval is shown. In $[15,16]$ the existence and stability of spikes is considered using formal asymptotic expansions. In [17, 18, 19] spikes are considered rigorously for the shadow system. In $[20,21]$ a skeleton structure and separators for the Gray-Scott model are established.

The structure of this paper is as follows:

In Section 2 we state and explain the main theorems on existence and stability.

In Section 3, we will prove the existence result, Theorem 2.1.

In Section 4, we provide some preliminary results on stability. In particular, we study the novel scalar nonlocal eigenvalue problem (NLEP) given in (4.3).

In Section 5, we separate the eigenvalue problem into two cases: small eigenvalues and large eigenvalues. The case of large eigenvalues is linked to a vectorial NLEP given in (5.4). This vectorial NLEP is then studied by reducing it to the scalar NLEP given in Section 4.

Appendix A contains a technical proof, namely that of Part (i) of Theorem 4.1.

Throughout this paper, the letter $C$ will denote various generic constants which are independent of $\epsilon$, for $\epsilon$ sufficiently small. The notation $A \sim B$ means that $\lim _{\epsilon \rightarrow 0} \frac{A}{B}=1$ and $A=O(B)$ is defined as $|A| \leq C|B|$ for some $C>0$.

2. Main Results: Existence and Stability. We now state the main results of this paper. We first construct stationary single-spike solutions to (1.1), i.e. singlespike solutions of the system

$$
\left\{\begin{array}{l}
D \Delta u_{i}+1-u_{i}-\frac{A}{\epsilon} u_{i} \sum_{j, k} W_{i}^{(j, k)} v_{j} v_{k}=0, \quad i=1, \ldots, M x \in \mathbb{R}, \\
\epsilon^{2} \Delta v_{i}-v_{i}+v_{i} \sum_{j, k=1} W_{j}^{(i, k)} u_{j} v_{k}=0, \quad i=1, \ldots, N, x \in \mathbb{R} .
\end{array}\right.
$$

In the case $M=N=2$, we will construct solutions of (2.1) which are even:

$$
\begin{gathered}
1-u_{i}=1-u_{i}(|x|) \in H^{1}(\mathbb{R}), \quad i=1,2, \\
v_{i}=v_{i}(|x|) \in H^{1}(R), \quad i=1,2 .
\end{gathered}
$$


Before stating the results, we need to introduce some assumptions and notations.

Let $w$ be the unique solution of the problem

$$
\left\{\begin{array}{l}
w_{y y}-w+w^{2}=0, w>0 \text { in } \mathbb{R} \\
w(0)=\max _{y \in \mathbb{R}} w(y), w(y) \rightarrow 0 \text { as }|y| \rightarrow+\infty
\end{array}\right.
$$

Note that (2.2) is an ODE problem and we can write $w$ explicitly as

$$
w(y)=\frac{3}{2 \cosh ^{2} \frac{y}{2}} .
$$

For the connection matrices we make the following three assumptions.

\section{Assumption 1:}

$$
\sum_{j, k} W_{j}^{(i, k)}=T, \quad i=1,2, \ldots, N \quad \text { for some } T>0 .
$$

In particular, (2.4) implies that $\sum_{j, k} W_{j}^{(i, k)}$ is independent of $i$.

We further assume

\section{Assumption 2:}

$$
\sum_{j, k} W_{i}^{(j, k)}=S, \quad i=1,2, \ldots, M \quad \text { for some } S>0 .
$$

In particular, (2.5) implies that $\sum_{j, k} W_{i}^{(j, k)}$ is independent of $i$.

The next assumption is the "transpose" of Assumption 1.

\section{Assumption 3:}

$$
\sum_{i, j} W_{j}^{(i, k)}=T
$$

In particular (2.6) implies that $\sum_{i, j} W_{j}^{(i, k)}$ is independent of $k$.

\section{Remark:}

Assumptions 1-3 state in a rigorous way that the interaction terms $W_{j}^{(i, k)}$, each of which couples two activators and one substrate, are balanced in the sense that the sum over two of these is independent of the third. For such a balanced system it is expected that solutions for which all components are non-vanishing do exist and can be stable. Two important cases are discussed below (see Case 1 and Case 2 on p.5).

We now state the existence result.

THEOREM 2.1.

Suppose that Assumptions 1-3 hold. Let $M=N=2$.

Assume that

$$
\epsilon<<1
$$

and

$$
\epsilon<<\frac{12 A S}{\sqrt{D} T^{2}}<1-\delta_{0} .
$$


(Expressed more precisely, (2.8) means the following: there are positive numbers $\delta_{0}$ and $\epsilon_{0}$ such that for all $\epsilon$ with $0<\epsilon<\epsilon_{0}$ we have $12 A S D^{-1 / 2} T^{-2}<1-\delta_{0}$; further, for all $\delta_{1}>0$ there exists $\epsilon_{0}>0$ such that $12 A S D^{-1 / 2} T^{-2}>\epsilon \delta_{1}$ for all $\epsilon$ with $0<\epsilon<\epsilon_{0}$.)

Then problem (2.1) admits two "single-spike" solutions $\left(u_{\epsilon}^{s}, v_{\epsilon}^{s}\right)=\left(u_{\epsilon, 1}^{s}, u_{\epsilon, 2}^{s}, v_{\epsilon, 1}^{s}, v_{\epsilon, 2}^{s}\right)$ and $\left(u_{\epsilon}^{l}, v_{\epsilon}^{l}\right)=\left(u_{\epsilon, 1}^{l}, u_{\epsilon, 2}^{l}, v_{\epsilon, 1}^{l}, v_{\epsilon, 2}^{l}\right)$ with the following properties:

(i) all components are even functions.

(ii) $u_{\epsilon, i}^{s}(x) \rightarrow 1 u_{\epsilon, i}^{l}(x) \rightarrow 1$ as $\epsilon \rightarrow 0$ for all $x \neq 0$ and $u_{\epsilon, i}^{s}(0), u_{\epsilon, i}^{l}(0)$ satisfy

$$
\begin{gathered}
u_{\epsilon, i}^{s}(0) \sim u^{s}, \quad u_{\epsilon, i}^{l}(0) \sim u^{l}, \quad i=1,2 \\
0<u^{s}<\frac{1}{2}<u^{l}<1 .
\end{gathered}
$$

(iii) $v_{\epsilon, i}^{s}=\xi^{s}(1+o(1)) w\left(\frac{|x|}{\epsilon}\right), \quad v_{\epsilon, i}^{l}=\xi^{s}(1+o(1)) w\left(\frac{|x|}{\epsilon}\right), \quad i=1,2$, as $\epsilon \rightarrow 0$ where $w$ is the unique solution of (2.2) and

$$
\xi^{s}=\frac{1}{S u^{s}}, \quad \xi^{l}=\frac{1}{S u^{l}}
$$

with $S$ defined in (2.5)

(iv) There exist $a>0, b>0$ such that

$$
\begin{aligned}
& 0<1-u_{\epsilon, i}^{s}(x) \leq C e^{-a|x|}, 0<1-u_{\epsilon, i}^{l}(x) \leq C e^{-a|x|}, \\
& 0<v_{\epsilon, i}^{s}(x) \leq C e^{-b \frac{|x|}{\epsilon}}, \quad 0<v_{\epsilon, i}^{l}(x) \leq C e^{-b \frac{|x|}{\epsilon}} .
\end{aligned}
$$

Finally, if $\epsilon$ is small enough and $12 A S D^{-1 / 2} T^{-2}>1+\delta_{0}$ (in the same sense as in (2.8)) then there are no single-spike solutions which satisfy (i) - (iv).

Theorem 2.1 will be proved in Section 3.

The second main goal of this paper is to study the stability properties of the spiky solution constructed in Theorem 2.1. We will show that, in agreement with the Gray-Scott model, the large solutions with $u_{\epsilon, i}(0) \sim u^{l}$ are always unstable [26, 27]. The small solutions with $u_{\epsilon, i}(0)=u^{s}$ can be linearly stable or unstable, depending on certain conditions for the parameters of the system (1.1). To elucidate this issue, we investigate their stability behavior in detail.

We say that a single-spike solution is linearly stable if the spectrum $\sigma\left(\mathcal{L}_{\epsilon}\right)$ of $\mathcal{L}_{\epsilon}$ (except for 0 ) lies in a left half plane $\left\{\lambda \in \mathcal{C}: \operatorname{Re}(\lambda)<-c_{0}\right\}$ for some $c_{0}>0$, and that 0 is a simple eigenvalue. A single-spike solution is called linearly unstable if there exists an eigenvalue $\lambda_{\epsilon}$ of $\mathcal{L}_{\epsilon}$ with $\operatorname{Re}\left(\lambda_{\epsilon}\right)>0$. Here $\mathcal{L}_{\epsilon}$ is the linearized operator around $\left(u_{\epsilon, i}, v_{\epsilon, j}\right)$ which will be defined in (5.1) below.

We now state our main result on stability.

Theorem 2.2. Suppose that Assumptions 1-3 hold. Let $M=N=2$. Assume that

$$
\epsilon<<1
$$

and

$$
\epsilon<<\frac{12 A S}{\sqrt{D} S T^{-2}}<1-\delta_{0},
$$


in the same sense as in (2.8).

Let

$\left(u_{\epsilon}^{s}, v_{\epsilon}^{s}\right)=\left(u_{\epsilon, 1}^{s}, u_{\epsilon, 2}^{s}, v_{\epsilon, 1}^{s}, v_{\epsilon, 2}^{s}\right)$ and $\left(u_{\epsilon}^{l}, v_{\epsilon}^{l}\right)=\left(u_{\epsilon, 1}^{l}, u_{\epsilon, 2}^{l}, v_{\epsilon, 1}^{l}, v_{\epsilon, 2}^{l}\right)$ be the solutions constructed in Theorem 2.1.

Let

$$
\begin{gathered}
a=\frac{1}{T} \sum_{j=1}^{2}\left(W_{j}^{(1,1)}-W_{j}^{(1,2)}\right) \\
b=\frac{1-u}{u \sqrt{1+\tau \lambda}+1-u} \frac{2}{T S} \sum_{j^{\prime}, k^{\prime}} W_{j^{\prime}}^{\left(1, k^{\prime}\right)}\left[W_{j^{\prime}}^{(1,1)}-W_{j^{\prime}}^{(2,2)}\right]
\end{gathered}
$$

where $u=u^{s}$ or $u=u^{l}$, respectively. Further, let

$$
b_{0}=\frac{2(1-u)}{T S} \sum_{j^{\prime}, k^{\prime}} W_{j^{\prime}}^{\left(1, k^{\prime}\right)}\left[W_{j^{\prime}}^{(1,1)}-W_{j^{\prime}}^{(2,2)}\right] .
$$

Then we have the following:

(1) (Stability) Assume that $b_{0}>a$. Suppose that $0 \leq \tau<\tau_{0}$, where $\tau_{0}>0$ may be chosen independently of $\epsilon$. Then $\left(u_{\epsilon}^{s}, v_{\epsilon}^{s}\right)=\left(u_{\epsilon, 1}^{s}, u_{\epsilon, 2}^{s}, v_{\epsilon, 1}^{s}, v_{\epsilon, 2}^{s}\right)$ is linearly stable.

(2) (Instability) Assume that $b_{0}<a$. Then $\left(u_{\epsilon}^{s}, v_{\epsilon}^{s}\right)=\left(u_{\epsilon, 1}^{s}, u_{\epsilon, 2}^{s}, v_{\epsilon, 1}^{s}, v_{\epsilon, 2}^{s}\right)$ is linearly unstable for all $\tau \geq 0$.

(3) (Instability) $\left(u_{\epsilon}^{l}, v_{\epsilon}^{l}\right)=\left(u_{\epsilon, 1}^{l}, u_{\epsilon, 2}^{l}, v_{\epsilon, 1}^{l}, v_{\epsilon, 2}^{l}\right)$ is linearly unstable for all $\tau \geq 0$. Theorem 2.2 will be proved in Sections 4 and 5 .

There are two important cases for which the conditions of Theorem 2.2 can be understood and interpreted very clearly in biological terms.

Case 1: Assume that

$$
W_{i}^{(j, k)} \text { is independent of } i \text { for } j, k=1,2 \text {. }
$$

which implies

$$
W_{j}^{(i, k)}=\frac{c+d}{2}+(-1)^{i-k} \frac{c-d}{2} \text { for some } c>0, d>0 .
$$

Then the condition $b_{0}>a$ in Theorem 2.2 takes the form $d>c$.

This means that the system can be stabilized if the off-diagonal interaction of the activators is dominated by their self-interaction.

\section{Case 2:}

We assume that

$$
W_{i}^{(j, k)}=0 \quad \text { if } j \neq k \text { for } i, j, k=1,2
$$

which implies

$$
W_{j}^{(i, k)}=\left(f+\left(f-\frac{S}{2}\right)(-1)^{i+j}\right) \delta_{i k} \quad \text { for some } f \text { with } S \geq f>0 .
$$


Then the condition $b_{0}>a$ in Theorem 2.2 takes the form

$$
0 \leq f<S\left(\frac{1}{2}-\frac{1}{2 \sqrt{2(1-u)}}\right) \quad \text { or } \quad S \geq f>S\left(\frac{1}{2}+\frac{1}{2 \sqrt{2(1-u)}}\right) .
$$

This means that the system can be stabilized if each activator has its preferred substrate with which it interacts more strongly that with the other.

3. Existence: Computation of the amplitudes. We will show the existence of solutions for which

$$
v_{\epsilon, i}(x)=(1+o(1)) \xi_{i} w\left(\frac{x}{\epsilon}\right), \quad \xi_{i}>0, \quad i=1,2
$$

where $\xi_{i}$ are positive constants. Substituting into (2.1), we see that the amplitudes $\xi_{i}$ necessarily have to satisfy

$$
\sum_{j, k=1}^{N} W_{j}^{(i, k)} u_{j}(0) \xi_{k}=1, \quad i=1,2 .
$$

From now on, we consider the special case $\xi_{i}=\xi$.

Proof of Theorem 2.1: Let $u_{\epsilon, i}(0) \sim u, i=1,2$. From (3.2), we get

$$
\sum_{j, k} W_{j}^{(i, k)} u \xi=1
$$

Together with Assumption 1, (3.3) implies

$$
T=\frac{1}{u \xi}
$$

To determine $u=\lim _{\epsilon \rightarrow 0} u_{\epsilon, i}(0)$, we use the Green's $G_{D}$ function defined by

$$
D G_{x x}(x, \xi)-G(x, \xi)+\delta_{\xi}=0 \quad \text { in } \mathbb{R}
$$

which is explicitly given by

$$
G(x, \xi)=\frac{1}{2 \sqrt{D}} e^{-|x-\xi| / \sqrt{D}} .
$$

This implies $G_{D}(0,0)=\frac{1}{2 \sqrt{D}}$.

We compute

$$
1-u=A G_{D}(0,0) \sum_{j, k} W_{i}^{(j, k)} \xi_{j} \xi_{k} u \int w^{2}
$$

Using Assumption 2, (3.4) and the relation $\int w^{2}=6$, we get

$$
1=u\left[1+6 A G_{D}(0,0) \sum_{j, k} W_{i}^{(j, k)} \xi_{j} \xi_{k} u\right]
$$




$$
=u\left[1+6 A G_{D}(0,0) S \frac{1}{u T^{2}}\right] .
$$

This implies

$$
u^{2}-u+6 A G_{D}(0,0) \frac{S}{T^{2}}=0
$$

The solutions are given by

$$
u=\frac{1}{2}\left(1 \pm \sqrt{1-\frac{24 A G_{D}(0,0) S}{T^{2}}}\right)=\frac{1}{2} \pm \sqrt{\frac{1}{4}-\frac{6 A G_{D}(0,0) S}{T^{2}}} .
$$

If $0<\frac{24 A G_{D}(0,0) S}{T^{2}}<1$, then there are two solutions for $u$ which are denoted as $u^{s}$ (with $0<u^{s}<\frac{1}{2}$ ) and $u^{l}$ (with $\frac{1}{2}<u^{l}<1$ ).

After $u^{s}$ and $u^{l}$ have been computed, the amplitudes $\xi^{s}$ and $\xi^{l}$ are derived from (3.2).

The proof of Theorem 2.1 goes along the same lines as in the proof of Theorem 2.1 of [27] or Theorem 1.1 of [28]. For completeness, we sketch the main steps.

First we express $u_{i}$ with $1-u_{i} \in H^{2}(\mathbb{R})$ in nonlocal form as $u_{i}=T_{i}[v]$, where $T_{i}[v]$ is the unique solution of the following linear equation:

$$
\Delta T_{i}[v]-T_{i}[v]-\frac{A}{\epsilon} T_{i}[v] \sum_{j, k} W_{i}^{(j, k)} v_{j} v_{k}=0 .
$$

Then, to construct a solution of (2.1), we look for functions

$$
v_{i}=u(0) w+\phi_{i, \epsilon}
$$

which satisfy the second set of equations in (2.1) (beginning with $\epsilon^{2} \Delta v_{i}-v_{i}+\ldots$ ), such that $\phi_{i, \epsilon}$ has a small norm in $H^{2}(\mathbb{R})$.

To this end, we have to study the linearized operator. The linearized operator has only the trivial kernel consisting of translation modes and is uniformly invertible after projections orthogonal to kernel and co-kernel. This result is given Theorem 5.1.

Now we can finish the existence proof by applying the contraction mapping principle.

4. Stability I: A nonlocal eigenvalue problem (NLEP). In this section, we will introduce and study a scalar nonlocal eigenvalue problem for later use.

Before stating the NLEP, we collect some known facts about the following eigenvalue problem which will be needed later:

$(\mathrm{EVP})$

$$
\left\{\begin{array}{c}
\Delta \phi-\phi+\lambda w \phi=0 \\
\phi \in H^{1}(\mathbb{R})
\end{array}\right.
$$

By Lemma 4.1 of [24], (EVP) admits the following set of eigenvalues

$$
\lambda_{1}=1, \lambda_{2}=2,2<\lambda_{3}<\lambda_{4} \leq \ldots .
$$

More precisely, by Appendix A of [29] the eigenvalues $\lambda_{n}$ are explicitly given by

$$
\lambda_{n}=\frac{(1+n)(2+n)}{6}, \quad n=1,2,3, \ldots, .
$$


THEOREM 4.1. We consider the nonlocal eigenvalue problem

$$
\Delta \phi-\phi+w \phi+a w \phi-b \frac{\int w \phi}{\int w^{2}} w^{2}=\lambda \phi .
$$

where $a>0$ and the complex number $b$ will be specified below.

(i) In the case $a>0$ and $b \geq 0$, (4.3) is stable (i.e. $\operatorname{Re}(\lambda) \leq-c$ for some $c>0$ independent of $\lambda$ ) if $b>a$ and $a<a^{*}<\frac{7}{3}$ for a certain constant $a^{*}>1$. It is unstable (i.e. there exists an eigenvalue $\lambda$ with $\operatorname{Re}(\lambda)>0$ ). if $b<a$.

(ii) In the case $a>0$ and $b=\frac{b_{0}}{u \sqrt{1+\tau \lambda}+1-u}$, (4.3) is stable if $a<a^{*}<\frac{7}{3}, b_{0}>a$ and $\tau$ is small enough. It is unstable for all $\tau \geq 0$ if $b_{0}<a$.

\section{Remarks:}

1. In the case $b=0$, then (4.3) is linearly stable if $a<0$ and linearly unstable if $a>0$. See Lemma 5.1 (3) of [29].

2. The NLEP (4.3) for $a=1$ and

$$
b=\frac{2(1-u)}{u \sqrt{1+\tau \lambda}+1-u}
$$

has been studied in Lemma 5.3 of [29]. It has been shown that for $\tau \geq 0$ and $\frac{1}{2}<u<1$ it has a positive eigenvalue.

3. By the definition of $a$ in Theorem 2.2 we have $|a| \leq 1$. Since $a^{*}>1$, for the original problem we always have $a<a^{*}$, the exact value of $a^{*}$ does not matter there.

4. For $a^{*}<a<7 / 3$ we have $h_{a}(0)<0$ and $h_{a}(0) \rightarrow-\infty$ as $\epsilon \rightarrow 7 / 3-$. Then instability depends on the size of $b$. The further we increase $a$, the larger $b$ must be chosen to avoid instability.

5. For $a>7 / 3$ we expect

$$
h_{a}(\alpha) \rightarrow \pm \infty \quad \text { as } \quad \alpha \rightarrow \mp \mu_{3}
$$

for some $0<\mu_{3}<\mu_{1}$. Then there must be a positive eigenvalue between $\mu_{3}$ and $\mu_{1}$ for any value of $b>0$.

Proof:

(i) We consider the linear operator

$$
L_{a} \phi:=\phi^{\prime \prime}-\phi+(a+1) w \phi
$$

where $0<a<\frac{7}{3}$. Note that for $a=\frac{7}{3}$ we get $a+1=\frac{10}{3}=\lambda_{3}$ which is the third eigenvalue of the eigenvalue problem (EVP) and the second eigenvalue of (EVP) in the class of even functions.

Then let

$$
\rho(a)=\int w L_{a}^{-1} w
$$

Claim 1: $\rho(1)>0$.

Proof: If $a=1$, then we compute

$$
L_{1} \phi=\phi^{\prime \prime}-\phi+2 w \phi
$$


and, as in [25], we derive

$$
L_{1}^{-1} w=w+\frac{x w^{\prime}}{2}
$$

Hence

$$
\rho(1)=\int w\left(w+\frac{x w^{\prime}}{2}\right)=\left(1-\frac{1}{4}\right) \int w^{2}>0 .
$$

Claim 2: $\rho^{\prime}(a)<0$ for $a<\frac{7}{3}$.

Proof: Let $L_{a}^{-1} w=: \phi_{a}$. Then

$$
\phi_{a}^{\prime \prime}-\phi_{a}+(a+1) w \phi_{a}=w .
$$

Consider $\psi=\frac{\partial \phi_{a}}{\partial a}$. Taking the derivative w.r.t. $a$ in (4.6), we get

$$
\psi^{\prime \prime}-\psi+(a+1) w \psi+w \phi_{a}=0
$$

Multiplying (4.7) by $\phi_{a}$ and (4.6) by $\psi$, we get

$$
\int w \psi=-\int w \phi_{a}^{2}
$$

This implies

$$
\rho^{\prime}(a)=\int w \frac{\partial \phi_{a}}{\partial a}=\int w \psi=-\int w \phi_{a}^{2}<0 .
$$

Claim 3: $\rho(a) \rightarrow-\infty$ as $a \rightarrow \frac{7}{3}$.

Proof: Let $\phi_{0}$ be the eigenfunction given by

$$
\phi_{0}^{\prime \prime}-\phi_{0}+\lambda_{3} w \phi_{0}=0 \text {. }
$$

Then we decompose

$$
\phi_{a}=\mu \phi_{0}+\phi_{a}^{\perp},
$$

where

$$
\int w \phi_{0} \phi_{a}^{\perp}=0
$$

We are now going to show that $\mu=c\left(a-\frac{7}{3}\right)^{-1}$ for some $c>0$ and $\phi_{a}^{\perp}=O(1)$ in $H_{r}^{2}(\mathbb{R})$. To this end, we derive the PDE for $\phi_{a}^{\perp}$ from (4.6) and (4.8):

$$
L_{a} \phi_{a}^{\perp}=w\left[1+\mu\left(\frac{7}{3}-a\right) \phi_{0}\right] .
$$

Multiplying (4.9) by $\phi_{0}$ and integrating, we get the solvability condition

$$
\left(a-\frac{7}{3}\right) \int w \phi_{0} \phi_{a}^{\perp}=\int w \phi_{0}-\left(a-\frac{7}{3}\right) \mu \int w \phi_{0}^{2}
$$


Using the definition of $\phi_{a}^{\perp}$, the l.h.s. in (4.10) vanishes. This implies

$$
\mu=\frac{\int w \phi_{0}}{\left(a-\frac{7}{3}\right) \int w \phi_{0}^{2}} .
$$

Using the invertibility of $L_{a}$, we derive

$$
\phi_{a}^{\perp}=O\left(1+\left|\frac{7}{3}-a\right| \mu\right) \quad \text { in } H_{r}^{2}(\mathbb{R}) .
$$

From (4.10), we get $\phi_{a}^{\perp}=O(1)$ in $H_{r}^{2}(\mathbb{R})$. We further derive

$$
\begin{gathered}
\rho(a)=\int w \phi_{a}=\mu \int w \phi_{0}+\int w \phi_{a}^{\perp} \\
=\frac{\left(\int w \phi_{0}\right)^{2}}{\left(a-\frac{7}{3}\right) \int w \phi_{0}^{2}}+\underbrace{\int w \phi_{a}^{\perp}}_{=O(1)}<0
\end{gathered}
$$

if $\int w \phi_{0} \neq 0,0<a<\frac{7}{3}$ and $a$ is sufficiently close to $7 / 3$.

We now show that in fact $\int w \phi_{0} \neq 0$. Suppose not, then $\int w \phi_{0}=0$. But then we also have $\int w \phi_{0}^{2}=0$ which is impossible. This is a contradiction and Claim 3 follows.

From Claim 1 - Claim 3, we conclude that there exists a unique $a^{*} \in\left(1, \frac{7}{3}\right)$ such that

$$
\int w L_{a}^{-1} w \begin{cases}>0 & \text { if } a<a^{*} \\ =0 & \text { if } a=a^{*} \\ <0 & \text { if } a>a^{*}\end{cases}
$$

The rest of the proof of Theorem 4.1 (i) follows the argument in [25]. For the convenience of the reader it is given in Appendix A.

Now we show Part (ii) of Theorem 4.1 following the proof of Lemma 5.3 of [29].

We show the stability part by a perturbation argument with respect to $\tau$ starting from $\tau=0$. We set $\lambda=\lambda_{R}+\sqrt{-1} \lambda_{I}$. We have show that $|\lambda| \leq C$ for some generic constant $C$ if $\lambda_{R} \geq 0$ and $0<\tau<1$. Multiplying (4.3) by the complex conjugate $\bar{\phi}$ of the eigenfunction and integrating, we can show that

$$
\left|\lambda_{I}\right| \leq C_{1}\left|\frac{b_{0}}{u \sqrt{1+\tau \lambda}+1-u}\right| \leq 2 C_{1}\left|b_{0}\right| .
$$

by considering the imaginary part of the resulting equation, where $C_{1}$ is independent of $\tau$. Taking the real part of the resulting equation, we get $\lambda_{R} \leq C_{2}$, where $C_{2}$ is independent of $\tau$. Therefore $|\lambda| \leq C_{3}$, where $C_{3}$ is independent of $\tau$, and the perturbation argument can be completed which implies stability.

To show the instability if $b_{0}<a$, we consider the function

$$
h_{a}(\alpha)=\int_{\mathbb{R}}\left(\left(L_{a}-\alpha\right)^{-1} w\right) w, \quad 0<\alpha<\mu_{1} .
$$


It is easy to see that

$$
\frac{d}{d \alpha} h_{a}(\alpha)=\int_{\mathbb{R}}\left(\left(L_{a}-\alpha\right)^{-2} w\right) w=\int_{\mathbb{R}}\left[\left(L_{a}-\alpha\right)^{-1} w\right]^{2}>0,
$$

and

$$
\lim _{\alpha \rightarrow \mu_{1}-} h_{a}(\alpha)=+\infty
$$

where $\mu_{1}$ is the unique positive eigenvalue of $L_{a}$. Next we consider the function

$$
g_{a}(\lambda)=\frac{u \sqrt{1+\tau \lambda}+1-u}{b_{0}}-\frac{1}{a}-\frac{\lambda}{a \int_{R} w^{2}} h_{a}(\lambda) .
$$

Note that

$$
g_{a}(0)=\frac{1}{b_{0}}-\frac{1}{a}>0
$$

On the other hand,

$$
\lim _{\lambda \rightarrow \mu_{1}-} g_{a}(\lambda)=-\infty
$$

Hence there must exist an $\lambda_{0} \in\left(0, \mu_{1}\right)$ such that $g_{a}\left(\lambda_{0}\right)=0$. This $\lambda_{0}>0$ is an eigenvalue of (4.3) which proves Part (ii) of Theorem 4.1.

\section{Stability II: Derivation of a vectorial NLEP and rigorous reduction.}

Linearizing (1.1) around the single-spike solution $u_{\epsilon, i}+\psi_{\epsilon, i} e^{\lambda t}, v_{\epsilon, j}+\phi_{\epsilon, j} e^{\lambda t}$, we study the eigenvalue problem of the linearized operator around $\left(u_{\epsilon, i}, v_{\epsilon, j}\right)$. We begin with the case of general positive integers $M$ and $N$.

The eigenvalue problem becomes

$$
\mathcal{L}_{\epsilon}\left(\begin{array}{c}
\psi_{\epsilon, j} \\
\phi_{\epsilon, i}
\end{array}\right)=\left(\begin{array}{c}
\lambda_{\epsilon} \tau \psi_{\epsilon, j} \\
\lambda_{\epsilon} \phi_{\epsilon, i}
\end{array}\right), i=1, \ldots, N, j=1, \ldots, M
$$

where $\lambda_{\epsilon} \in \mathcal{C}$, the set of complex numbers.

By taking derivatives of (2.1) it follows that 0 is an eigenvalue of $\mathcal{L}_{\epsilon}$ with eigenfuction $u_{\epsilon, i}^{\prime}, v_{\epsilon, j}^{\prime}$.

To prove Theorem 2.2, we first derive from (5.1) that

$\left\{\begin{array}{l}\Delta \phi_{\epsilon, i}-\phi_{\epsilon, i}+\phi_{\epsilon, i} \sum_{j, k} W_{j}^{(i, k)} u_{\epsilon, j} v_{\epsilon, k}+v_{\epsilon, i} \sum_{j, k} W_{j}^{(i, k)}\left(u_{\epsilon, j} \phi_{\epsilon, k}+\psi_{\epsilon, j} v_{\epsilon, k}\right)=\lambda_{\epsilon} \phi_{\epsilon, i}, \\ \Delta \psi_{\epsilon, i}-\psi_{\epsilon, i}-\frac{A}{\epsilon} \psi_{\epsilon, i} \sum_{j, k} W_{i}^{(j, k)} v_{\epsilon, j} v_{\epsilon, k}-\frac{A}{\epsilon} u_{\epsilon, i} \sum_{j, k} W_{i}^{(j, k)}\left(\phi_{\epsilon, j} v_{\epsilon, k}+v_{\epsilon, j} \phi_{\epsilon, k}\right)=\tau \lambda_{\epsilon} \psi_{\epsilon, i} .\end{array}\right.$

We assume that the domain of $\mathcal{L}_{\epsilon}$ is $\left(H^{2}(\mathbb{R})\right)^{N}$. This is possible since we can express $\psi_{\epsilon, i} \in H^{2}(\mathbb{R})$ in nonlocal form as $\psi_{\epsilon, i}=T_{i}^{\prime}\left[v_{\epsilon}, \phi_{\epsilon}\right]$, where $T_{i}^{\prime}\left[v_{\epsilon}, \phi_{\epsilon}\right]$ satisfies the following linear equation, which has a unique solution:

$$
\Delta T_{i}^{\prime}\left[v_{\epsilon}, \phi_{\epsilon}\right]-\left(1+\tau \lambda_{\epsilon}\right) T_{i}^{\prime}\left[v_{\epsilon}, \phi_{\epsilon}\right]-\frac{A}{\epsilon} T_{i}^{\prime}\left[v_{\epsilon}, \phi_{\epsilon}\right] \sum_{j, k} W_{i}^{(j, k)} v_{\epsilon, j} v_{\epsilon, k}
$$




$$
-\frac{A}{\epsilon} u_{\epsilon, i} \sum_{j, k} W_{i}^{(j, k)}\left(\phi_{\epsilon, j} v_{\epsilon, k}+v_{\epsilon, j} \phi_{\epsilon, k}\right)=0 .
$$

The eigenvalue problem (5.2) implies (from now on dropping the index $\epsilon$ )

$$
\begin{gathered}
\psi_{i}(0)=-A G_{D}^{*}(0,0) \psi_{i}(0) \sum_{j, k} W_{i}^{(j, k)} \xi^{2} \int w^{2} \\
-A G_{D}^{*}(0,0) u \xi \sum_{j, k} W_{i}^{(j, k)} \int\left(\phi_{j}+\phi_{k}\right) w+o(2),
\end{gathered}
$$

where

$$
G_{D}(0,0)=\frac{\theta}{2}, \quad G_{D}^{*}(0,0)=\frac{\theta}{2 \sqrt{1+\tau \lambda}} .
$$

This implies

$$
\begin{gathered}
\psi_{i}(0)=-\frac{A G_{D}^{*}(0,0) u \xi \sum_{j, k} W_{i}^{(j, k)} \int\left(\phi_{j}+\phi_{k}\right) w}{1+6 A G_{D}^{*}(0,0) \xi^{2} \sum_{j, k} W_{i}^{(j, k)}}+o(1) \\
=-\frac{A u \frac{\theta}{2} \xi^{2} \sum_{j, k} W_{i}^{(j, k)} \int\left(\phi_{j}+\phi_{k}\right) w}{(\sqrt{1+\tau \lambda}-1)+\frac{1}{u}+o(1)} .
\end{gathered}
$$

Taking the limit $\epsilon \rightarrow 0$, this leads to the NLEP

$$
\begin{gathered}
\Delta \phi_{i}-\phi_{i}+\phi_{i} w+\frac{\sum_{k} \phi_{k} \sum_{j} W_{j}^{(i, k)}}{\sum_{j, k} W_{j}^{(i, k)}} w \\
-\frac{1-u}{u \sqrt{1+\tau \lambda}+1-u} \frac{\sum_{j^{\prime}, k^{\prime}} W_{j^{\prime}}^{\left(i, k^{\prime}\right)} \sum_{j, k} W_{j^{\prime}}^{(j, k)} \int\left(\phi_{j}+\phi_{k}\right) w}{\sum_{j^{\prime}, k^{\prime}} W_{j^{\prime}}^{\left(i, k^{\prime}\right)} \sum_{j, k} W_{j^{\prime}}^{(j, k)}} \frac{w^{2}}{\int w^{2}}=\lambda \phi_{i}, \quad i=1, \ldots, N .
\end{gathered}
$$

Considering this NLEP, we see that the factors

$$
\frac{\sum_{j} W_{j}^{(i, k)}}{\sum_{j, k} W_{j}^{(i, k)}}, \quad k=1, \ldots, N
$$

and

$$
\frac{\sum_{j^{\prime}, k^{\prime}} W_{j^{\prime}}^{\left(i, k^{\prime}\right)} W_{j^{\prime}}^{(j, k)}}{\sum_{j^{\prime}, k^{\prime}} W_{j^{\prime}}^{\left(i, k^{\prime}\right)} \sum_{j, k} W_{j^{\prime}}^{(j, k)}}, \quad j, k=1, \ldots, N
$$

are both weighted averages. Using the notation $T$ and $S$, introduced in (2.4) and (2.5), respectively, these factors can be rewritten as

$$
\frac{1}{T} \sum_{j} W_{j}^{(i, k)}, \quad k=1, \ldots, N
$$


and

$$
\frac{1}{S T} \sum_{j^{\prime}, k^{\prime}} W_{j^{\prime}}^{\left(i, k^{\prime}\right)} W_{j^{\prime}}^{(j, k)}, \quad j, k=1, \ldots, N
$$

Then the NLEP (5.3) becomes

$$
\Delta \phi_{i}-\phi_{i}+\phi_{i} w+\frac{1}{T} \sum_{k} \phi_{k} \sum_{j} W_{j}^{(i, k)} w
$$

$-\frac{1-u}{u \sqrt{1+\tau \lambda}+1-u} \frac{1}{S T} \sum_{j^{\prime}, k^{\prime}} W_{j^{\prime}}^{\left(i, k^{\prime}\right)} \sum_{j, k} W_{j^{\prime}}^{(j, k)} w^{2} \frac{\int\left(\phi_{j}+\phi_{k}\right) w}{\int w^{2}}=\lambda \phi_{i}, \quad i=1, \ldots, N$.

Although the derivations given above are formal, we can rigorously prove the following separation of eigenvalues in the special case $M=N=2$. We set

$$
\begin{gathered}
a=\frac{1}{T} \sum_{j, k=1}^{2}(-1)^{k+1} W_{j}^{(1, k)} \\
=\frac{1}{T} \sum_{j=1}^{2}\left(W_{j}^{(1,1)}-W_{j}^{(1,2)}\right), \\
b=\frac{1-u}{u \sqrt{1+\tau \lambda}+1-u} \frac{1}{T S} \sum_{j^{\prime}=1}^{2} \sum_{k^{\prime}=1}^{2} W_{j^{\prime}}^{\left(1, k^{\prime}\right)}\left[\sum_{j=1}^{2} \sum_{k=1}^{2} W_{j^{\prime}}^{(j, k)}\left((-1)^{j}+(-1)^{k}\right)\right] \\
=\frac{1-u}{u \sqrt{1+\tau \lambda}+1-u} \frac{2}{T S} \sum_{j^{\prime}=1}^{2} \sum_{k^{\prime}=1}^{2} W_{j^{\prime}}^{\left(1, k^{\prime}\right)}\left[W_{j^{\prime}}^{(1,1)}-W_{j^{\prime}}^{(2,2)}\right] .
\end{gathered}
$$

TheOREM 5.1. Let $M=N=2$. Suppose that $0<a<a^{*}$ and $b \neq a$.

Let $\lambda_{\epsilon}$ be an eigenvalue of (5.2) for which $\operatorname{Re}\left(\lambda_{\epsilon}\right)>-a_{0}$.

(1) Suppose that (for suitable sequences $\epsilon_{n} \rightarrow 0$ ) we have $\lambda_{\epsilon_{n}} \rightarrow 0$ as $n \rightarrow \infty$. Then for $n$ sufficiently large, it follows that $\lambda_{\epsilon_{n}}=0$ and

$$
\left(\phi_{\epsilon_{n}, 1}, \phi_{\epsilon_{n}, 2}, \psi_{\epsilon_{n}, 1}, \psi_{\epsilon_{n}, 2}\right) \in \operatorname{span}\left\{u_{\epsilon_{n}, 1}^{\prime}, u_{\epsilon_{n}, 2}^{\prime}, v_{\epsilon_{n}, 1}^{\prime}, v_{\epsilon_{n}, 2}^{\prime}\right\} .
$$

The operator $\mathcal{L}_{\epsilon}$ is invertible if restricted as follows

$$
\mathcal{L}_{\epsilon}: \mathcal{K}_{\epsilon}^{\perp, 1} \rightarrow \mathcal{K}_{\epsilon}^{\perp, 2},
$$

where

$$
\begin{aligned}
& \mathcal{K}_{\epsilon}^{\perp, 1}=\left\{u \in\left(H^{2}(\mathbb{R})\right)^{2} \mid \int_{\mathbb{R}} u_{i} u_{\epsilon_{n}, i}^{\prime}=0, i=1,2\right\} \oplus\left(H^{2}(\mathbb{R})\right)^{2}, \\
& \mathcal{K}_{\epsilon}^{\perp, 2}=\left\{u \in\left(L^{2}(\mathbb{R})\right)^{2} \mid \int_{\mathbb{R}} u_{i} u_{\epsilon_{n}, i}^{\prime}=0, i=1,2\right\} \oplus\left(H^{2}(\mathbb{R})\right)^{2},
\end{aligned}
$$


where $\oplus$ is used to denote the direct product of two spaces.

(2) Suppose that (for suitable sequences $\epsilon_{n} \rightarrow 0$ ) we have $\lambda_{\epsilon_{n}} \rightarrow \lambda_{0} \neq 0$. Then $\lambda_{0}$ is an eigenvalue of the NLEP given in (5.4).

(3) Let $\lambda_{0} \neq 0$ be an eigenvalue of the NLEP given in (5.4). Then for $\epsilon$ sufficiently small, there is an eigenvalue $\lambda_{\epsilon}$ of (5.2) with $\lambda_{\epsilon} \rightarrow \lambda_{0}$ as $\epsilon \rightarrow 0$.

From Theorem 5.1 we see rigorously that the eigenvalue problem (5.2) is reduced to the study of the vectorial NLEP (5.4).

Now we prove Theorem 5.1.

\section{Proof of Theorem 5.1:}

For the proof of (1), we proceed as in the proof of Theorem 2.2 (3) in [27], where existence and stability of single-spike states for the Gray-Scott system in 2-D are studied. The proof can be adapted to our system by considering the 1-D situation as in [29]. Then Theorem 5.1 (1) follows from Lemma 5.2, by the same proof as for Theorem 2.2 (3) of [27]. Next we state and prove Lemma 5.2 which concludes the proof of Theorem 5.1 (1).

Let us denote the linear operator on the left hand side of (5.4) as $\mathcal{L}$, where $\mathcal{L}:\left(H^{2}(\mathbb{R})\right)^{2} \rightarrow\left(L^{2}(\mathbb{R})\right)^{2}$. Then we have

Lemma 5.2.

Assume that $0<a<a^{*}$ and $b \neq a$.

(1). Let $\phi$ be an eigenfunction of (5.4) with $\lambda_{0}=0$. Then we have

$$
\phi \in \mathcal{K}_{0}:=\operatorname{span}\left\{w^{\prime}(y) \vec{e}_{0}\right\},
$$

where $\vec{e}_{0}=(1,1)^{\tau}$. (This implies that $\operatorname{Ker}(\mathcal{L})=\mathcal{K}_{0}$.)

(2). The operator $\mathcal{L}$ is invertible if restricted as follows

$$
\mathcal{L}: \mathcal{K}_{0}^{\perp, 1} \rightarrow \mathcal{K}_{0}^{\perp, 2},
$$

where

$$
\begin{aligned}
& \mathcal{K}_{0}^{\perp, 1}=\left\{u \in\left(H^{2}(\mathbb{R})\right)^{2} \mid \int_{\mathbb{R}} u w^{\prime}(y) \overrightarrow{e_{0}}=0\right\}, \\
& \mathcal{K}_{0}^{\perp, 2}=\left\{u \in\left(L^{2}(\mathbb{R})\right)^{2} \mid \int_{\mathbb{R}} u w^{\prime}(y) \overrightarrow{e_{0}}=0\right\} .
\end{aligned}
$$

\section{Proof:}

The proof of Lemma 5.2 (1) follows by adding the equations and integrating which implies that $\phi_{1}+\phi_{2}=0$. Then, integrating the equation for $\phi_{1}$, it follows that $\phi_{1}=0$.

The proof of Lemma 5.2 (2) is based on showing that the adjoint operator has only the trivial kernel (translation modes). This is done by multiplication with $w$ followed by integration and then using the same arguments as before.

For the details we refer to [32].

Theorem 5.1 (2) is a consequence of the asymptotic analysis performed at the beginning of this section.

To prove Theorem 5.1 (3), we follow the argument given in Section 2 of [1] to show that if $\lambda_{0} \neq 0$ is an unstable eigenvalue of a limiting eigenvalue problem, then, 
for $\epsilon$ small enough, there exists an eigenvalue $\lambda_{\epsilon}$ of the finite $\epsilon$ problem such that $\lambda_{\epsilon} \rightarrow \lambda_{0}$. We now adapt that argument to the current problem.

Let $\lambda_{0} \neq 0$ be an eigenvalue of problem (5.4) with $\operatorname{Re}\left(\lambda_{0}\right)>0$. We first note that from the linear equations for $\psi_{\epsilon, i}$, we can express $\psi_{\epsilon, i}$ in terms of $\left(\phi_{\epsilon, 1}, \phi_{\epsilon, 2}\right)$. Then we write the equation for $\phi_{\epsilon, i}$ as follows:

$$
\phi_{\epsilon, i}=R_{\epsilon}\left(\lambda_{\epsilon}\right)\left[\phi_{\epsilon, i} \sum_{j, k} W_{j}^{(i, k)} u_{j} v_{k}+v_{i} \sum_{j, k} W_{j}^{(i, k)}\left(u_{j} \phi_{\epsilon, k}+\psi_{\epsilon, j} v_{k}\right)\right],
$$

where $i=1, \ldots, N$ and $R_{\epsilon}(\lambda)$ is the inverse of $-\Delta+\left(1+\lambda_{\epsilon}\right)$ in $H^{2}(\mathbb{R})$ (which exists if $\operatorname{Re}\left(\lambda_{\epsilon}\right)>-1$ or $\left.\operatorname{Im}\left(\lambda_{\epsilon}\right) \neq 0\right)$. The crucial fact is that $R_{\epsilon}\left(\lambda_{\epsilon}\right)$ is a compact operator if $\epsilon$ is sufficiently small. The rest of the proof follows that in [1] and the details are omitted.

The proof of Theorem 5.1 is completed.

We now conclude the proof of Theorem 2.1.

Adding the equations in (5.4), we get the following NLEP for $\phi=\sum_{k} \phi_{k}$ :

$$
\Delta \phi-\phi+2 w \phi-2 \frac{1-u}{u \sqrt{1+\tau \lambda}+1-u} \frac{\int w \phi}{\int w^{2}} w^{2}=\lambda \phi .
$$

The nonlocal term in (5.9) has been derived as follows: We computed the first $\operatorname{sum} \sum_{i, k^{\prime}} W_{j^{\prime}}^{\left(i, k^{\prime}\right)}=S$. The second sum gives

$$
\begin{gathered}
\sum_{j^{\prime}, j, k} W_{j^{\prime}}^{(j, k)} \int\left(\phi_{j}+\phi_{k}\right) w \\
=\sum_{j^{\prime}, j, k} W_{j^{\prime}}^{(j, k)} \int \phi_{j} w+\sum_{j^{\prime}, j, k} W_{j^{\prime}}^{(j, k)} \int \phi_{k} w \\
=\sum_{j} \sum_{j^{\prime}, k} W_{j^{\prime}}^{(j, k)} \int \phi_{j} w+\sum_{k} \sum_{j^{\prime}, j} W_{j^{\prime}}^{(j, k)} \int \phi_{k} w \\
=\sum_{j} T \int \phi_{j} w+\sum_{k} T \int \phi_{k} w \\
=2 T \int \phi w .
\end{gathered}
$$

We now study the stability properties of (5.9).

Assuming that $\operatorname{Re}(\lambda)>0$ and $u=u^{s}$, then for $\tau=0$ or $\tau$ small enough, it follows from Theorem 4.1 (i) that $\phi=\phi_{1}+\phi_{2}+\cdots+\phi_{N}=0$.

We now consider the special case $M=N=2$, i.e. we assume that there are two activators and two substrates. Then we get $\phi_{1}=-\phi_{2}$, where $\phi_{1}$ has to satisfy the eigenvalue problem

$$
\Delta \phi_{1}-\phi_{1}+w \phi_{1}+a w \phi_{1}-b \frac{\int w \phi_{1}}{\int w^{2}} w^{2}=\lambda \phi_{1},
$$


introduced in (4.3), where

$$
\begin{gathered}
a=\frac{1}{T} \sum_{j, k=1}^{2}(-1)^{k+1} W_{j}^{(1, k)} \\
=\frac{1}{T} \sum_{j=1}^{2}\left(W_{j}^{(1,1)}-W_{j}^{(1,2)}\right), \\
b=\frac{1-u}{u \sqrt{1+\tau \lambda}+1-u} \frac{1}{T S} \sum_{j^{\prime}=1}^{2} \sum_{k^{\prime}=1}^{2} W_{j^{\prime}}^{\left(1, k^{\prime}\right)}\left[\sum_{j=1}^{2} \sum_{k=1}^{2} W_{j^{\prime}}^{(j, k)}\left((-1)^{j}+(-1)^{k}\right)\right] \\
=\frac{1-u}{u \sqrt{1+\tau \lambda}+1-u} \frac{2}{T S} \sum_{j^{\prime}=1}^{2} \sum_{k^{\prime}=1}^{2} W_{j^{\prime}}^{\left(1, k^{\prime}\right)}\left[W_{j^{\prime}}^{(1,1)}-W_{j^{\prime}}^{(2,2)}\right] .
\end{gathered}
$$

(Recall that $a$ and $b$ have been defined in (5.5) and (5.6), respectively.)

Remark: For general $M$ and $N$, NLEP (4.3) is replaced by the vectorial NLEP

$$
\begin{gathered}
\Delta \phi_{i}-\phi_{i}+\phi_{i} w+\frac{1}{T} \sum_{k=1}^{N-1} \phi_{k} \sum_{j=1}^{M}\left(W_{j}^{(i, k)}-W_{j}^{(i, N)}\right) w \\
-\frac{1-u}{u \sqrt{1+\tau \lambda}+1-u} \frac{1}{S T} \sum_{k^{\prime}=1}^{N} \sum_{j^{\prime}=1}^{M} W_{j^{\prime}}^{\left(i, k^{\prime}\right)} \\
\sum_{k=1}^{N-1}\left(\sum_{j=1}^{N-1}\left(W_{j^{\prime}}^{(j, k)}+W_{j^{\prime}}^{(k, j)}\right)-\sum_{j \neq k, N}\left(W_{j^{\prime}}^{(j, N)}+W_{j^{\prime}}^{(N, j)}\right)-2 W_{j^{\prime}}^{(N, N)}\right) \frac{\int \phi_{k} w}{\int w^{2}} w^{2} \\
=\lambda \phi_{i}, \quad i=1, \ldots, N-1 .
\end{gathered}
$$

Again, invoking Theorem 4.1, in the case of real constants $a>0$ and $b \geq 0$, (4.3) is stable if $a<a^{*}<\frac{7}{3}$ and $b>a$ for a certain constant $a^{*}$. In the case $a>0$ and $b=\frac{b_{0}}{u \sqrt{1+\tau \lambda}+1-u},(4.3)$ is stable if $a<a^{*}<\frac{7}{3}, b_{0}>a$ and $\tau$ is small enough. Therefore, under either of these sets of conditions, we derive $\phi_{1}=\phi_{2}=0$. This proves that stability.

To prove the instability, we have to construct pairs $\left(\phi_{1}, \phi_{2}\right)$ of unstable eigenfunctions for (5.4).

If $b<a$ or $b=\frac{b_{0}}{u \sqrt{1+\tau \lambda}+1-u}$ with $b_{0}<a$, we set $\left(\phi_{1}, \phi_{2}\right)=\left(\phi_{0},-\phi_{0}\right)$, where $\phi_{0}$ is the eigenfunction of (4.3) with largest (positive) eigenvalue. Then $\left(\phi_{1}, \phi_{2}\right)$ is an unstable eigenfunction of (5.4) which has the same eigenvalue.

If $u=u^{l}$, where $\frac{1}{2}<u^{l}<1$, then we set $\left(\phi_{1}, \phi_{2}\right)=\left(\phi_{0}, 0\right)$, where $\phi_{0}$ is the eigenfunction of (4.3) with $a=1$ and $b=1-u$ or $b_{0}=1-u$ which has the largest 
(positive) eigenvalue. The eigenfunction $\phi_{0}$ exists by Remark 2 after Theorem 4.1. Then $\left(\phi_{1}, \phi_{2}\right)$ is an unstable eigenfunction (5.4) which has the same eigenvalue.

This concludes the proof of Theorem 2.2 .

We now consider the stability in some important special situations.

First, in the case of real constants $a$ and $b$, we study if the main condition $b>a$ in Theorem 2.2 is true in the following two important cases:

\section{Case 1:}

We assume as in (2.12) that

$$
W_{i}^{(j, k)} \text { is independent of } i \text { for } j, k=1,2 .
$$

The four conditions in (2.12) imply that Assumption 2, given in (2.5), holds. Using Assumption 1 (2.4) and Assumption 3 (2.6), we get

$$
W_{j}^{(i, k)}=\frac{c+d}{2}+(-1)^{i-k} \frac{c-d}{2} \text { for some } c>0, d>0 .
$$

Note that $W_{j}^{(i, k)}$ is symmetric in $i, k$. Further, $c$ are the diagonal terms and $d$ the off-diagonal terms of $W_{j}^{(i, k)}$ in $i, k$. Then for the constants in Theorem 2.2 we get

$$
a=\frac{c-d}{c+d}, \quad b=0 .
$$

Then the condition $b>a$ holds iff $d>c$.

Note that for $d>c$ the NLEP (4.3) is stable even for any $\tau \geq 0$. However, to prove the stability of (5.4), we have to take into account an extra threshold for $\tau$ coming from (5.9). We will come back to this issue below and indicate a numerical method to compute this threshold based on hypergeometric functions.

This means that we have stability if the off-diagonal interactions for the activators dominate.

A similar behavior has been observed in the special case $M=1$. In particular, for the hypercycle, for which in the connection matrix the only non-vanishing elements are $W_{1}^{1, N}$ and $W_{1}^{k, k-1}(k=2, \ldots, N)$, it has been shown that stable spikes are possible $[28,29]$.

\section{Case 2:}

We assume as in (2.13) that

$$
W_{i}^{(j, k)}=0 \quad \text { if } j \neq k \text { for } i, j, k=1,2 .
$$

These four conditions together with Assumption 1 (2.4) and Assumption 2 (2.5) imply

$$
W_{j}^{(i, k)}=\left(f+\left(f-\frac{S}{2}\right)(-1)^{i+j}\right) \delta_{i k} \quad \text { for some } f \text { with } S \geq f>0 .
$$

We further derive that in this case we necessarily have $S=T$ and that Assumption 3 (2.6) is satisfied.

We note that in contrast to Case 1 there are no off-diagonal terms with $j \neq k$ for the connection matrices. This means that the activators do not show any crossinteraction, only self-interaction is allowed. Our goal now is to find out if the selfinteraction terms alone are able to stabilize the system leading to stable configurations 
of spikes. This is a new effect which has not been considered in any previous work. Now the case $\tau>0$ can only be considered numerically, whereas the case $\tau=0$ is easier and allows a closed analytical solution. Therefore we consider $\tau=0$ first.

For $\tau=0$ we get the NLEP in (4.3) with $a=1$ and

$$
\begin{gathered}
b=\frac{2}{S T}\left[W_{1}^{(1,1)}\left(W_{1}^{(1,1)}-W_{1}^{(2,2)}\right)+W_{2}^{(1,1)}\left(W_{2}^{(1,1)}-W_{2}^{(2,2)}\right)\right](1-u) \\
=2\left(1-\frac{2 f}{S}\right)^{2}(1-u) .
\end{gathered}
$$

We have stability iff $b>1$ which is equivalent to

$$
0 \leq f<S\left(\frac{1}{2}-\frac{1}{2 \sqrt{2(1-u)}}\right) \quad \text { or } \quad S \geq f>S\left(\frac{1}{2}+\frac{1}{2 \sqrt{2(1-u)}}\right) .
$$

This result can be interpreted as follows: The spike is stable if each of the activators has its own preferred substrate. In particular, this is true in the following two extreme cases:

In the first extreme case, $f=1$, the first activator interacts only with the first substrate and the second activator only with the second substrate.

In the second extreme case, $f=0$, the first activator interacts only with the second substrate and the second activator only with the first substrate.

In both of these extreme cases the system is decomposed into two separate GrayScott systems for which stability has been shown before [13, 14, 30, 31].

The result in this case shows that the preference of activators towards their own substrates must be above a certain threshold to imply stability of the spike.

The result can be interpreted as the robustness of the stability of several coupled Gray-Scott systems which are stable individually under the assumption that the coupling is not too strong.

Such an effect does not occur for hypercycles since for them there is only one substrate.

Finally, we consider Case 2 with general $\tau>0$. We get (4.3) with $a=1$ and

$$
b=\frac{2}{S T}\left[W_{1}^{(1,1)}\left(W_{1}^{(1,1)}-W_{1}^{(2,2)}\right)+W_{2}^{(1,1)}\left(W_{2}^{(1,1)}-W_{2}^{(2,2)}\right)\right] \frac{1-u}{u \sqrt{1+\tau \lambda}+1-u}
$$

$$
=2\left(1-\frac{2 f}{S}\right)^{2} \frac{1-u}{u \sqrt{1+\tau \lambda}+1-u} .
$$

Using the strategy in [29], we derive the following result which will allows us to compute the eigenvalue of (4.3) numerically for $\tau>0$.

Let us first introduce the so-called generalized Gauss function. Let $a_{1}, a_{2}, \ldots, a_{A}$ and $b_{1}, b_{2}, \ldots, b_{B}$ be two sequences of numbers. Consider the series

$$
1+\frac{a_{1} a_{2} \ldots a_{A}}{b_{1} b_{2} \ldots b_{B}} \frac{z}{1 !}+\frac{\left(a_{1}+1\right)\left(a_{2}+1\right) \ldots\left(a_{A}+1\right)}{\left(b_{1}+1\right)\left(b_{2}+1\right) \ldots\left(b_{B}+1\right)} \frac{z^{2}}{2 !}+\ldots
$$




$$
\equiv{ }_{A} F_{B}\left\{\begin{array}{ccccc}
a_{1}, & a_{2}, & \ldots, & a_{A} & ; \\
b_{1}, & b_{2}, & \ldots, & b_{B} & ;
\end{array}\right\}
$$

${ }_{A} F_{B}$ is called generalized Gauss function or generalized hypergeometric function. For more details on such functions, we refer to [22].

Then we have the following lemma.

LEMma 5.3. Let $\lambda$ be an eigenvalue of problem (4.3) with $b$ given by (5.11) and $\operatorname{Re}(\lambda) \geq 0$. Then $\lambda$ is a solution of the algebraic equation

$$
\begin{aligned}
& \frac{u \sqrt{1+\tau \lambda}+1-u}{2(1-2 f / S)^{2}(1-u)} a-1 \\
& =-{ }_{4} F_{3}\left\{\begin{array}{lllll}
1, & 3, & -\frac{1}{2}, & 2 & ; \\
2+\gamma, & 2-\gamma, & \frac{5}{2} & ;
\end{array}\right\} \\
& +\frac{\lambda}{3} B_{1} \frac{\Gamma(1+\gamma) \Gamma\left(\frac{5}{2}\right)}{\Gamma\left(\gamma+\frac{3}{2}\right)}{ }_{3} F_{2}\left\{\begin{array}{rccc}
2+\gamma, & \gamma-\frac{3}{2}, & 1+\gamma & ; \\
1+2 \gamma, & \frac{3}{2}+\gamma & ;
\end{array}\right\}
\end{aligned}
$$

where $\gamma=\sqrt{1+\lambda}$ and $B_{1}$ is given by (5.16) below.

Proof: We give a sketch of the proof. For more details, we refer to [29] and [12]. Let $\phi$ be the unique solution of

$$
\Delta \phi-\gamma^{2} \phi+(1+a) w \phi=w, \quad \phi^{\prime}(0)=0, \phi \in H^{1}(\mathbb{R}) .
$$

Then it is easy to see that (4.3) is equivalent to (compare (4.13):

$$
\lambda \frac{\int w \phi}{\int w^{2}}=\frac{a}{b}-1
$$

where

$$
b=\frac{2(1-2 f / S)^{2}(1-u)}{u \sqrt{1+\tau \lambda}+1-u} .
$$

Now we compute $\phi$. As in [29], we make a change of dependent variables: Let

$$
z=\frac{2}{3} w(y)
$$

Note that $z(y)$ is one-to-one with $z \rightarrow 0$ as $y \rightarrow \infty$ and $z \rightarrow 1$ as $y \rightarrow 0$.

Then by the results of [29] and [12], we obtain

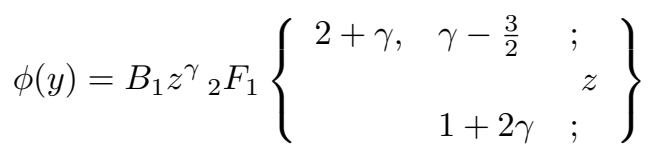

$$
\begin{aligned}
& +\frac{3}{2} \frac{1}{1-\gamma^{2}} z_{3} F_{2}\left\{\begin{array}{cccc}
1, & 3, & -\frac{1}{2} & ; \\
2+\gamma, & 2-\gamma & ;
\end{array}\right\},
\end{aligned}
$$


where the constant $B_{1}$ is given by

$$
B_{1}=-\frac{3}{2} \frac{1}{1-\gamma^{2}} \frac{(\Gamma(2+\gamma))^{2}}{\Gamma(3) \Gamma(1+2 \gamma)} \frac{\Gamma\left(\gamma-\frac{3}{2}\right) \Gamma(2-\gamma)}{\Gamma\left(-\frac{1}{2}\right) \Gamma(1)} .
$$

Using the following integral property, whose proof can be found in [22]:

$$
\begin{aligned}
& { }_{A+1} F_{B+1}\left\{\begin{array}{cccccc}
a_{1}, & a_{2}, & \ldots, & a_{A}, & c, & ; \\
b_{1}, & b_{2}, & \ldots, & b_{B}, & d & ;
\end{array}\right\} \\
& =\frac{\Gamma(d)}{\Gamma(c) \Gamma(d-c)} \int_{0}^{1} t^{c-1}(1-t)^{d-c-1}{ }_{A} F_{B}\left\{\begin{array}{ccccc}
a_{1}, & a_{2}, & \ldots, & a_{A} & ; \\
& & & & \\
b_{1}, & b_{2}, & \ldots, & b_{B} & ;
\end{array}\right\} d t
\end{aligned}
$$

we compute that

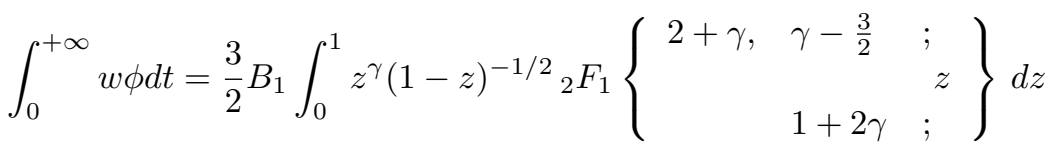

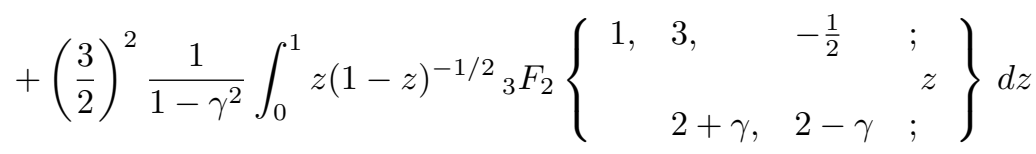

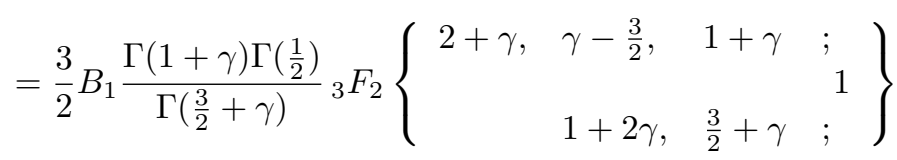

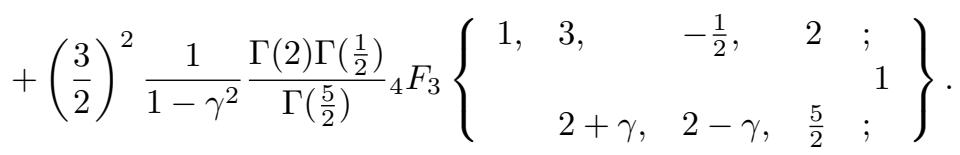

Finally, using the formula

$$
\int_{0}^{+\infty} w^{2} d t=\left(\frac{3}{2}\right)^{2} \int_{0}^{1} z^{2}(1-z)^{-\frac{1}{2}} d z=\left(\frac{3}{2}\right)^{2} \frac{\Gamma(2) \Gamma\left(\frac{1}{2}\right)}{\Gamma\left(\frac{5}{2}\right)}
$$

and substituting into (5.15), we obtain (5.13).

By Lemma 5.3, problem (4.3) can be solved by using Mathematica. This method can also be adapted to study the stability in Case 1 for $\tau>0$. We will not produce any numerical results here. The readers are referred to [2] for some numerical results in the case $M=N=1$.

Acknowledgments: This research is supported by an Earmarked Research Grant from RGC of Hong Kong. MW thanks the Department of Mathematics at The Chinese University of Hong Kong for their kind hospitality. 
6. Appendix A: Proof of Part (i) of Theorem 4.1. Note that the operator $L_{n}$ defined by

$$
L_{n} \phi=L_{a} \phi-a \frac{\int w \phi}{\int w^{2}} w^{2}, \quad \phi \in H_{r}^{1}(\mathbb{R})
$$

is not self-adjoint, where $H_{r}^{1}(\mathbb{R})$ is the linear subspace of even functions within $H^{1}(\mathbb{R})$. Let $0<a<\frac{7}{3}$. (The case $a=1$ has been studied in [25].) Then we have $X_{a}:=$ $\operatorname{kernel}\left(L_{a}\right) \cap H_{r}^{1}(\mathbb{R})=\{0\}$. Further,

$$
L_{a} w=a w^{2} .
$$

Since $L_{n}$ is not self-adjoint, we introduce a new operator $L$ which is defined as follows:

$$
L \phi:=L_{a} \phi-a \frac{\int_{\mathbb{R}} w \phi}{\int_{\mathbb{R}} w^{2}} w^{2}-a \frac{\int_{\mathbb{R}} w^{2} \phi}{\int_{\mathbb{R}} w^{2}} w+a \frac{\int_{\mathbb{R}} w^{3} \int_{\mathbb{R}} w \phi}{\left(\int_{\mathbb{R}} w^{2}\right)^{2}} w .
$$

We have the following important lemma.

LEMMA 6.1. (1) The operator $L$ is self-adjoint and the kernel $X$ of $L$ within $H_{r}^{1}(\mathbb{R})$ is given by $X=\operatorname{span}\{w\}$.

(2) There exists a positive constant $a_{1}>0$ such that

$$
\begin{gathered}
Q[\phi]:=-\int_{\mathbb{R}} \phi(L \phi)=\int_{\mathbb{R}}\left(|\nabla \phi|^{2}+\phi^{2}-(a+1) w \phi^{2}\right) \\
+\frac{2 a \int_{\mathbb{R}} w \phi \int_{\mathbb{R}} w^{2} \phi}{\int_{\mathbb{R}} w^{2}}-a \frac{\int_{\mathbb{R}} w^{3}}{\left(\int_{\mathbb{R}} w^{2}\right)^{2}}\left(\int_{\mathbb{R}} w \phi\right)^{2} \\
\geq a_{1} d_{L^{2}(\mathbb{R})}^{2}(\phi, X)
\end{gathered}
$$

for all $\phi \in H_{r}^{1}(\mathbb{R})$, where $d_{L^{2}(\mathbb{R})}$ means the distance in $L^{2}$-norm.

Proof of Lemma 6.1:

By (6.2), $L$ is self-adjoint.

Next we compute the kernel of $L$. It is easy to see that $w \in \operatorname{kernel}(L)$.

On the other hand, if $\phi \in \operatorname{kernel}(L)$, then by $(6.1)$

$$
L_{a} \phi=c_{1}(\phi) w+c_{2}(\phi) w^{2}=c_{1}(\phi) w+c_{2}(\phi) L_{0}\left(\frac{w}{a}\right)
$$

where

$$
c_{1}(\phi)=a \frac{\int_{\mathbb{R}} w^{2} \phi}{\int_{\mathbb{R}} w^{2}}-a \frac{\int_{\mathbb{R}} w^{3} \int_{\mathbb{R}} w \phi}{\left(\int_{\mathbb{R}} w^{2}\right)^{2}}, \quad c_{2}(\phi)=a \frac{\int_{\mathbb{R}} w \phi}{\int_{\mathbb{R}} w^{2}} .
$$

Hence

$$
\phi-c_{1}(\phi) L_{a}^{-1} w-c_{2}(\phi) \frac{1}{a} w \in \operatorname{kernel}\left(L_{a}\right) .
$$

Substituting (6.4) into (6.3), we derive

$$
c_{1}(\phi)=a c_{1}(\phi) \frac{\int_{\mathbb{R}} w^{2} L_{a}^{-1} w}{\int_{\mathbb{R}} w^{2}}-a c_{1}(\phi) \frac{\int_{\mathbb{R}} w^{3} \int_{\mathbb{R}} w L_{a}^{-1} w}{\left(\int_{\mathbb{R}} w^{2}\right)^{2}}
$$




$$
\begin{gathered}
=a c_{1}(\phi) \frac{\int_{\mathbb{R}}\left(L_{a}^{-1} w^{2}\right) w}{\int_{\mathbb{R}} w^{2}}-a c_{1}(\phi) \frac{\int_{\mathbb{R}} w^{3} \int_{\mathbb{R}} w L_{a}^{-1} w}{\left(\int_{\mathbb{R}} w^{2}\right)^{2}} \\
=c_{1}(\phi)-a c_{1}(\phi) \frac{\int_{\mathbb{R}} w^{3} \int_{\mathbb{R}} w L_{a}^{-1} w}{\left(\int_{\mathbb{R}} w^{2}\right)^{2}} .
\end{gathered}
$$

This implies that $c_{1}(\phi)=0$. By (6.4) and since $\operatorname{kernel}\left(L_{a}\right)=\{0\}$, Part (1) follows.

It remains to prove Part (2). Suppose Part (2) is not true. Then, by Part (1), there exists $(\alpha, \phi)$ such that (i) $\alpha$ is real and positive, (ii) $\phi \in H_{r}^{1}(\mathbb{R}), \phi \perp w$, and (ii) $L \phi=\alpha \phi$.

We show that this is impossible. From (ii) and (iii), we have

$$
\left(L_{a}-\alpha\right) \phi=a \frac{\int_{\mathbb{R}} w^{2} \phi}{\int_{\mathbb{R}} w^{2}} w .
$$

We first claim that $\int_{\mathbb{R}} w^{2} \phi \neq 0$. In fact if $\int_{\mathbb{R}} w^{2} \phi=0$, then $\alpha>0$ is an eigenvalue of $L_{a}$. By the properties of $L_{a}, \alpha=\mu_{1}$ is the principle eigenvalue of $L_{a}$ and $\phi$ has constant sign. This contradicts the fact that $\phi \perp w$. Therefore $\alpha \neq \mu_{1}$, and hence $L_{a}-\alpha$ is invertible in $H_{r}^{1}(\mathbb{R})$. So $(6.5)$ implies

$$
\phi=a \frac{\int_{\mathbb{R}} w^{2} \phi}{\int_{\mathbb{R}} w^{2}}\left(L_{a}-\alpha\right)^{-1} w .
$$

Thus

$$
\begin{gathered}
\int_{\mathbb{R}} w^{2} \phi=a \frac{\int_{\mathbb{R}} w^{2} \phi}{\int_{\mathbb{R}} w^{2}} \int_{\mathbb{R}}\left(\left(L_{a}-\alpha\right)^{-1} w\right) w^{2}, \\
\int_{\mathbb{R}} w^{2}=a \int_{\mathbb{R}}\left(\left(L_{a}-\alpha\right)^{-1} w\right) w^{2}, \\
\int_{\mathbb{R}} w^{2}=\int_{\mathbb{R}}\left(\left(L_{a}-\alpha\right)^{-1} w\right)\left(\left(L_{a}-\alpha\right) w+\alpha w\right), \\
0=\int_{\mathbb{R}}\left(\left(L_{a}-\alpha\right)^{-1} w\right) w .
\end{gathered}
$$

Let $h_{a}(\alpha)=\int_{\mathbb{R}}\left(\left(L_{a}-\alpha\right)^{-1} w\right) w$. Then $h_{a}(0)=\rho(a)=\int_{\mathbb{R}}\left(L_{a}^{-1} w\right) w>0$ if $0<a<a^{*}$.

Moreover $h_{a}^{\prime}(\alpha)=\int_{\mathbb{R}}\left(\left(L_{a}-\alpha\right)^{-2} w\right) w=\int_{\mathbb{R}}\left(\left(L_{a}-\alpha\right)^{-1} w\right)^{2}>0$. This implies $h_{a}(\alpha)>0$ for all $\alpha \in\left(0, \mu_{1}\right)$. Clearly, also $h_{a}(\alpha)<0$ for $\alpha \in\left(\mu_{1}, \infty\right)$ (since $\left.\lim _{\alpha \rightarrow+\infty} h_{a}(\alpha)=0\right)$. Therefore there can be no solution of $h_{a}(\alpha)=0$. This is a contradiction to (6.6). This finishes the proof of Lemma 6.1.

Now we finish the proof of Theorem 4.1 (i).

Let $\alpha_{0}=\alpha_{R}+i \alpha_{I}$ and $\phi=\phi_{R}+i \phi_{I}$ be an eigenvalue and an eigenfunction of the NLEP (4.3). Then we obtain the two equations

$$
L_{a} \phi_{R}-b \frac{\int_{\mathbb{R}} w \phi_{R}}{\int_{\mathbb{R}} w^{2}} w^{2}=\alpha_{R} \phi_{R}-\alpha_{I} \phi_{I},
$$




$$
L_{a} \phi_{I}-b \frac{\int_{\mathbb{R}} w \phi_{I}}{\int_{\mathbb{R}} w^{2}} w^{2}=\alpha_{R} \phi_{I}+\alpha_{I} \phi_{R} .
$$

Multiplying (6.7) by $\phi_{R}$ and (6.8) by $\phi_{I}$, integrating and adding the two equations, we get

$$
\begin{gathered}
-\alpha_{R} \int_{\mathbb{R}}\left(\phi_{R}+\phi_{I}^{2}\right)=Q\left[\phi_{R}\right]+Q\left[\phi_{I}\right] \\
+(b-2 a) \frac{\int_{\mathbb{R}} w \phi_{R} \int_{\mathbb{R}} w^{2} \phi_{R}+\int_{\mathbb{R}} w \phi_{I} \int_{\mathbb{R}} w^{2} \phi_{I}}{\int_{\mathbb{R}} w^{2}} \\
+a \frac{\int_{\mathbb{R}} w^{3}}{\left(\int_{\mathbb{R}} w^{2}\right)^{2}}\left[\left(\int_{\mathbb{R}} w \phi_{R}\right)^{2}+\left(\int_{\mathbb{R}} w \phi_{I}\right)^{2}\right] .
\end{gathered}
$$

Multiplying both (6.7) and (6.8) by $w$, we obtain after integration

$$
\begin{aligned}
& a \int_{\mathbb{R}} w^{2} \phi_{R}-b \frac{\int_{\mathbb{R}} w \phi_{R}}{\int_{\mathbb{R}} w^{2}} \int_{\mathbb{R}} w^{3}=\alpha_{R} \int_{\mathbb{R}} w \phi_{R}-\alpha_{I} \int_{\mathbb{R}} w \phi_{I}, \\
& a \int_{\mathbb{R}} w^{2} \phi_{I}-b \frac{\int_{\mathbb{R}} w \phi_{I}}{\int_{\mathbb{R}} w^{2}} \int_{\mathbb{R}} w^{3}=\alpha_{R} \int_{\mathbb{R}} w \phi_{I}+\alpha_{I} \int_{\mathbb{R}} w \phi_{R} .
\end{aligned}
$$

Multiplying (6.9) by $\int_{\mathbb{R}} w \phi_{R}$ and (6.10) by $\int_{\mathbb{R}} w \phi_{I}$ and adding the equations, we get

$$
\begin{gathered}
a \int_{\mathbb{R}} w \phi_{R} \int_{\mathbb{R}} w^{2} \phi_{R}+a \int_{\mathbb{R}} w \phi_{I} \int_{\mathbb{R}} w^{2} \phi_{I} \\
=\left(\alpha_{R}+b \frac{\int_{\mathbb{R}} w^{3}}{\int_{\mathbb{R}} w^{2}}\right)\left(\left(\int_{\mathbb{R}} w \phi_{R}\right)^{2}+\left(\int_{\mathbb{R}} w \phi_{I}\right)^{2}\right) .
\end{gathered}
$$

This implies

$$
\begin{gathered}
-\alpha_{R} \int_{\mathbb{R}}\left(\phi_{R}+\phi_{I}^{2}\right)=Q\left[\phi_{R}\right]+Q\left[\phi_{I}\right] \\
+(b-2 a)\left(\frac{1}{a} \alpha_{R}+\frac{b}{a} \frac{\int_{\mathbb{R}} w^{3}}{\int_{\mathbb{R}} w^{2}}\right) \frac{\left(\int_{\mathbb{R}} w \phi_{R}\right)^{2}+\left(\int_{\mathbb{R}} w \phi_{I}\right)^{2}}{\int_{\mathbb{R}} w^{2}} \\
+a \frac{\int_{\mathbb{R}} w^{3}}{\left(\int_{\mathbb{R}} w^{2}\right)^{2}}\left[\left(\int_{\mathbb{R}} w \phi_{R}\right)^{2}+\left(\int_{\mathbb{R}} w \phi_{I}\right)^{2}\right] .
\end{gathered}
$$

Set

$$
\phi_{R}=c_{R} w+\phi_{R}^{\perp}, \phi_{R}^{\perp} \perp w, \quad \phi_{I}=c_{I} w+\phi_{I}^{\perp}, \phi_{I}^{\perp} \perp w .
$$


Then

$$
\begin{gathered}
\int_{\mathbb{R}} w \phi_{R}=c_{R} \int_{\mathbb{R}} w^{2}, \int_{\mathbb{R}} w \phi_{I}=c_{I} \int_{\mathbb{R}} w^{2}, \\
d_{L^{2}(\mathbb{R})}^{2}\left(\phi_{R}, X\right)=\left\|\phi_{R}^{\perp}\right\|_{L^{2}(\mathbb{R})}^{2}, \quad d_{L^{2}(\mathbb{R})}^{2}\left(\phi_{I}, X\right)=\left\|\phi_{I}^{\perp}\right\|_{L^{2}(\mathbb{R})}^{2} .
\end{gathered}
$$

After some elementary computations, we get

$$
Q\left[\phi_{R}\right]+Q\left[\phi_{I}\right]
$$

$+\left(\frac{b}{a}-1\right) \alpha_{R}\left(c_{R}^{2}+c_{I}^{2}\right) \int_{\mathbb{R}} w^{2}+a\left(\frac{b}{a}-1\right)^{2}\left(c_{R}^{2}+c_{I}^{2}\right) \int_{\mathbb{R}} w^{3}+\alpha_{R}\left(\left\|\phi_{R}^{\perp}\right\|_{L^{2}}^{2}+\left\|\phi_{I}^{\perp}\right\|_{L^{2}}^{2}\right)=0$.

By Lemma 6.1 (2), we have

$$
\begin{gathered}
\left(\frac{b}{a}-1\right) \alpha_{R}\left(c_{R}^{2}+c_{I}^{2}\right) \int_{\mathbb{R}} w^{2} \\
+a\left(\frac{b}{a}-1\right)^{2}\left(c_{R}^{2}+c_{I}^{2}\right) \int_{\mathbb{R}} w^{3}+\left(\alpha_{R}+a_{1}\right)\left(\left\|\phi_{R}^{\perp}\right\|_{L^{2}}^{2}+\left\|\phi_{I}^{\perp}\right\|_{L^{2}}^{2}\right) \leq 0 .
\end{gathered}
$$

Since $\frac{b}{a}>1$, we must have $\alpha_{R}<0$. This proves Part (i) of Theorem 4.1.

\section{REFERENCES}

[1] E.N. Dancer, On stability and Hopf bifurcations for chemotaxis systems, to appear in Methods Appl. Anal. (2001).

[2] A. Doelman, A. Gardner and T.J. Kaper, Stability analysis of singular patterns in the 1-D Gray-Scott model: A matched asymptotic approach, Phys. D 122 (1998), 1-36.

[3] A. Doelman, A. Gardner and T.J. Kaper, A stability index analysis of 1-D patterns of the Gray-Scott model, Methods Appl. Anal. 7 (2000).

[4] A. Doelman, T. Kaper and P. A. Zegeling, Pattern formation in the one-dimensional Gray-Scott model, Nonlinearity 10 (1997), 523-563.

[5] A. Doelman, R. Gardner and T. J. Kaper, Large stable pulse solutions in reaction-diffusion equations, Indiana Univ. Math. J. 50, 443-507 (2001).

[6] M. Eigen and P. Schuster, The hypercycle. A principle of natural self organisation. Part A. Emergence of the hypercycle. Naturwissenschaften 64 (1977), 541-565.

[7] M. Eigen and P. Schuster, The hypercycle. A principle of natural self organisation. Part B. The abstract hypercycle. Naturwissenschaften 65 (1978), 7-41.

[8] M. Eigen and P. Schuster, The hypercycle. A principle of natural self organisation. Part C. The realistic hypercycle. Naturwissenschaften 65 (1978), 341-369.

[9] M. Eigen and P. Schuster, The hypercycle: A principle of natural selforganisation (Springer, Berlin, 1979).

[10] P. Gray and S.K. Scott, Autocatalytic reactions in the isothermal, continuous stirred tank reactor: isolas and other forms of multistability, Chem. Eng. Sci. 38 (1983), 29-43.

[11] P. Gray and S.K. Scott, Autocatalytic reactions in the isothermal, continuous stirred tank reactor: oscillations and instabilities in the system $A+2 B \rightarrow 3 B, B \rightarrow C$, Chem. Eng. Sci. 39 (1984), 1087-1097.

[12] T. Kolokolnikov and J. Wei, Stability of spiky solutions in a competition model with crossdiffusion, preprint. 
[13] T. Kolokolnikov, M.J. Ward and J. Wei, The existence and stability of spike equilibria in the one-dimensional Gray-Scott model: the low feed-rate regime, Stud. Appl. Math. 115 (2005), 21-71.

[14] T. Kolokolnikov, M.J. Ward and J. Wei, The existence and stability of spike equilibria in the one-dimensional Gray-Scott model: the pulse-splitting regime, Phys. D 202 (2005), 258-293.

[15] C.B. Muratov, V.V. Osipov, Static spike autosolitons in the Gray-Scott model, J. Phys. A 33 (2000), 8893-8916.

[16] C.B. Muratov, V.V. Osipov, Stability of the static spike autosolitons in the Gray-Scott model, SIAM J. Appl. Math. 62 (2002), 1463-1487

[17] W.-M. Ni and I. Takagi, On the shape of least energy solution to a semilinear Neumann problem, Comm. Pure Appl. Math. 41 (1991), 819-851.

[18] W.-M. Ni and I. Takagi, Locating the peaks of least energy solutions to a semilinear Neumann problem, Duke Math. J. 70 (1993), 247-281.

[19] W.-M. Ni and I. Takagi, Point-condensation generated by a reaction-diffusion system in axially symmetric domains, Japan J. Industrial Appl. Math. 12 (1995), 327-365.

[20] Y. Nishiura and D. Ueyama, A skeleton structure of self-replicating dynamics, Physcia D 130 (1999), 73-104.

[21] Y. Nishiura, T. Teramoto and D. Ueyama, Scattering and separators in dissipative systems, Phys. Rev. E 67 (2003), 056210.

[22] L.J. Slater, Generalized hypergeometric functions, Cambridge University Press, 1966.

[23] H. Takagi and K. Kaneko, Differentiation and replication of spots in a reaction-diffusion systesm with many chemicals, Europhys. Lett. 56 (2001), 145-151.

[24] J. Wei, On the construction of single-peaked solutions to a singularly perturbed semilinear Dirichlet problem, J. Differential Equations 129 (1996), 315-333.

[25] J. Wei, On single interior spike solutions of Gierer-Meinhardt system: uniqueness, spectrum estimates and stability analysis, Euro. J. Appl. Math. 10 (1999), 353-378.

[26] J. Wei, Existence, stability and metastability of point condensation patterns generated by Gray-Scott system, Nonlinearity 12 (1999), 593-616.

[27] J. Wei, On two dimensional Gray-Scott model: existence of single pulse solutions and their stability, Phys. D, 148 (2001), 20-48.

[28] J. Wei and M. Winter, On a two dimensional reaction-diffusion system with hypercyclical structure, Nonlinearity 13 (2000), 2005-2032.

[29] J. Wei and M. Winter, Critical threshold and stability of cluster solutions for large reactiondiffusion systems in $\mathbb{R}^{1}$, SIAM J. Math Anal. 33 (2002), 1058-1089.

[30] J. Wei and M. Winter, Existence and stability of multiple-spot solutions for the Gray-Scott model in $\mathbb{R}^{2}$, Phys. D 176 (2003), 147-180.

[31] J. Wei and M. Winter, Asymmetric spotty patterns for the Gray-Scott model in $\mathbb{R}^{2}$, Stud. Appl. Math. 110 (2003), 63-102.

[32] J. Wei and M. Winter, Existence, classification and stability analysis of multiple-peaked solutions for the Gierer-Meinhardt system in $\mathbb{R}^{1}$, Methods Appl. Anal. 14 (2007), 119-164. 